INPLASY

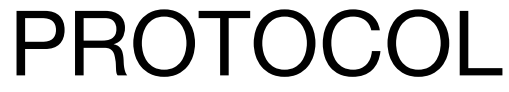

To cite: Cao et al. Mental

Fatigue and Basketball

Performance: A Systematic

Review. Inplasy protocol

2021100017. doi:

10.37766/inplasy2021.10.0017

Received: 05 October 2021

Published: 05 October 2021

Corresponding author:

Shudian Cao

caoshudian0516@163.com

Author Affiliation:

University Putra Malaysia

Physical Education.

Support: No support.

Review Stage at time of this submission: Data extraction Completed but not published.

Conflicts of interest:

None declared.

\section{Mental Fatigue and Basketball Performance: A Systematic Review}

Cao, S1; Soh, K².

Review question / Objective: Does mental fatigue impair basketball performance?

Condition being studied: Mental Fatigue and Basketball Performance. The PRISMA table has been down. And we find 6 articles that meet the inclusion criteria. Now, we are analysing these articles.

Eligibility criteria: (1) an evaluation of basketball-specific tests performed after the mental fatigue (MF)-inducing intervention was required, and, in the control group, the MF should not have been induced, or at least have triggered less MF than the intervention task; (2) the intervention was used to induce MF, and the sample population was comprised of basketball players; (3) measurements were implemented in a basketballspecific context (i.e. jump shots, dribbling, and passing); (4) the outcomes encompassed any form of basketball performance; and (5) randomized controlled trials (RCTs), non-randomized controlled trials (nRCTs), and nonrandomized non-controlled trials (nRnCTs) had to be included.

INPLASY registration number: This protocol was registered with the International Platform of Registered Systematic Review and Meta-Analysis Protocols (INPLASY) on 05 October 2021 and was last updated on 05 October 2021 (registration number INPLASY2021100017).

\section{INTRODUCTION}

Review question / Objective: Does mental fatigue impair basketball performance?

Condition being studied: Mental Fatigue and Basketball Performance. The PRISMA table has been down. And we find 6 articles that meet the inclusion criteria. Now, we are analysing these articles.

\section{METHODS}

Participant or population: Basketball players (all sexes). 
Intervention: Inclusion criteria: The intervention was targeted to induce mental fatigue, such as Stroop task, in which participants were required to verbalize as quickly and accurately as possible the ink color of the words while ignoring the word content. The task was set up so that participants performed five 3-min blocks each, consisting of 135 trials, separated by four 30-s breaks. It is a usually way to induce mental fatigue.Exclusion criteria: The intervention does not induce mental fatigue successfully.

Comparator: Inclusion criteria: All the control conditions that would not induce mental fatigue, such as watching documentaries.Exclusion criteria: The control conditions that have the possibility to induce mental fatigue.

Study designs to be included: RCTs, nRCTs, and $\mathrm{nRnCTs.}$

Eligibility criteria: (1) an evaluation of basketball-specific tests performed after the mental fatigue (MF)-inducing intervention was required, and, in the control group, the MF should not have been induced, or at least have triggered less MF than the intervention task; (2) the intervention was used to induce MF, and the sample population was comprised of basketball players; (3) measurements were implemented in a basketball-specific context (i.e. jump shots, dribbling, and passing); (4) the outcomes encompassed any form of basketball performance; and (5) randomized controlled trials (RCTs), nonrandomized controlled trials (nRCTs), and non-randomized non-controlled trials (nRnCTs) had to be included.

Information sources: PubMed, Web of Science, SPORTDiscus, Scopes, and China National Knowledge Infrastructure (CNKI).

Main outcome(s): Reviewing the articles on mental fatigue in basketball and finding the effects of mental fatigue on basketball performance. Trying to figure out the recovery strategies to help participants reduce mental fatigue.
Quality assessment / Risk of bias analysis: 'QualSyst' was used to assess the methodology quality (Kmet \& Lee, 2004). It contained 14 items. The score was set according to the degree to which certain criteria were met (yes $=2$, partial $=1$, no $=$ $0)$. 'NA' was marked when the items did not apply to the study design and excluded from the total calculation of the score. A score of $\geq 75 \%$ indicated strong quality, a score of $55-75 \%$ indicated moderate quality, and a score of $\leq 55 \%$ indicated weak quality.

Strategy of data synthesis: 1. For synthesis, the outcomes that encompassed any form of basketball performance required, such as physical, tactical, technical, or cognitive performance. 2. After the intervention, whether the mental fatigue is induced should be known. 3. An evaluation of the basketball-specific tests after the mental fatigue inducing intervention was required.

Subgroup analysis: Study design: RCTs, nRCTs, and nRnCTs; Intervention: mental fatigue; Setting: basketball court; Participant characteristics: male, minor or adult.

Sensitivity analysis: The results (titles and/ or abstracts) of studies retrieved using the search strategy and the titles and/or abstracts of studies from other sources will be independently screened by two review authors to identify studies that may meet the above inclusion criteria. The reviewers will review these studies according to the standard of population, intervention, controversion, outcome, and study design. The two review authors will extract data independently, and the differences will be determined and resolved through discussion (discuss with the third author if necessary). The results (titles and/or abstracts) of studies retrieved using the search strategy and the titles and/or abstracts of studies from other sources will be independently screened by two review authors to identify studies that may meet the above inclusion criteria. The reviewers will review these studies according to the standard of population, intervention, controversion, outcome, and study design. 
The two review authors will extract data independently, and the differences will be determined and resolved through discussion (discuss with the third author if necessary).

Country(ies) involved: China.

Keywords: Mental Fatigue; Sports; Athletic Performance; Basketball.

Contributions of each author:

Author 1 - Shudian Cao - The author drafted the manuscript.

Email: caoshudian0516@163.com

Author 2 - Kim Geok Soh - The author provided a lot of advice.

Email: kims@upm.edu.my 